\title{
Bismuth Redox-Catalyzed Fluorination of Arylboron Reagents
}

\section{Key words}

fluorination

bismuth

redox catalysis
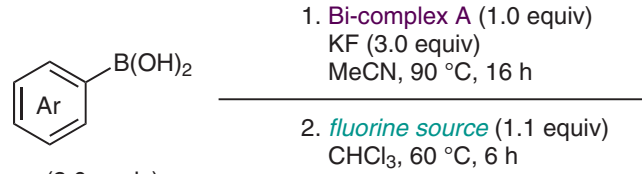

(2.0 equiv) $\mathrm{CHCl}_{3}, 60{ }^{\circ} \mathrm{C}, 6 \mathrm{~h}$

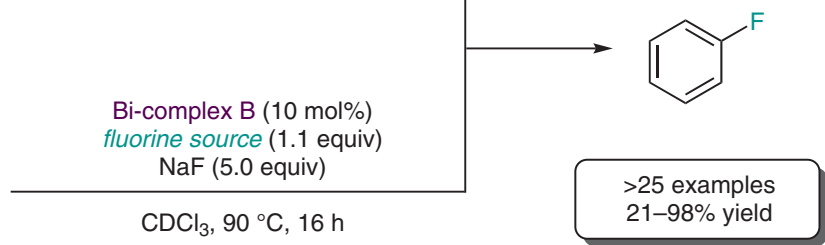

(2.0 equiv)

Proposed mechanism:
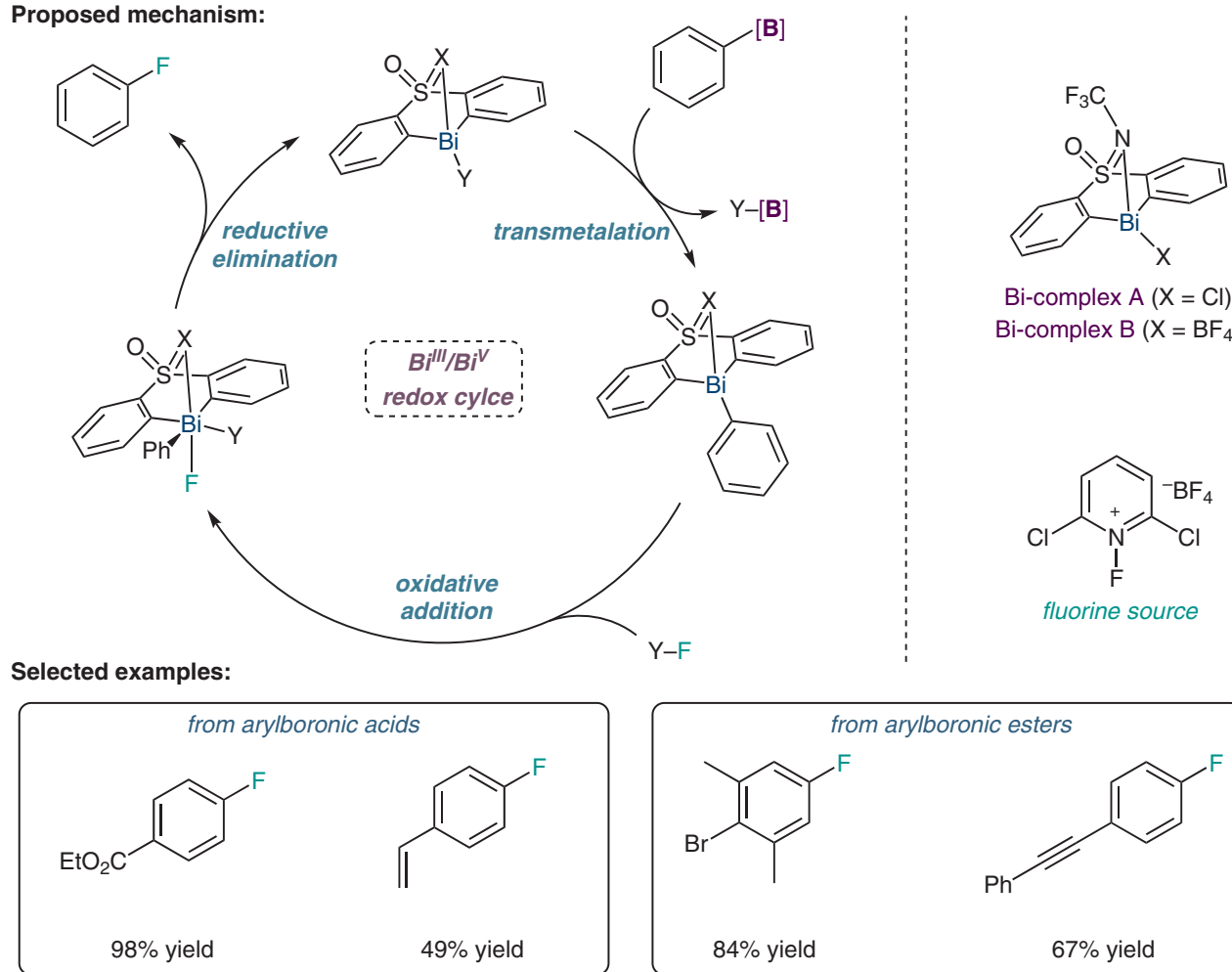

Bi-complex $\mathrm{A}(\mathrm{X}=\mathrm{Cl})$

Bi-complex $\mathrm{B}\left(\mathrm{X}=\mathrm{BF}_{4}\right)$

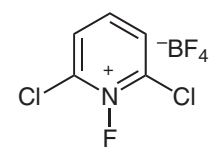

fluorine source

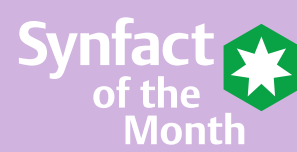

Significance: The authors report a bismuth redox-catalyzed fluorination of functionalized arylboronic esters or acids. The key role is played by the newly designed bismuth complex, which can change oxidation state and therefore undergo both oxidative addition and reductive elimination like a transition metal.
Comment: Based on crystallographic characterization of the bismuth species involved, combined with mechanistic investigations, Cornella and coworkers revealed the presence of a $\mathrm{Bi}(\mathrm{III}) / \mathrm{Bi}(\mathrm{V})$ redox couple, which has never been observed before. This work demonstrates the possibility of giving non- $d$-block elements transition-metal-like behavior by careful tuning of the ligand. 